\title{
Iron deficiency from the standpoint of cardiac rehabilitation: novel therapeutic opportunities
}

\author{
Gabriella Malfatto ${ }^{1}$, Anna Frisinghelli ${ }^{2}$, Marco Ambrosetti ${ }^{3}$, Maria Teresa La Rovere ${ }^{4}$, Irene Motta $^{5}$, \\ Bruno Passaretti ${ }^{6}$, Marco Pavesi ${ }^{7}$, Roberto Tramarin ${ }^{8}$, Nicolò Dasseni ${ }^{9}$, Pompilio Faggiano ${ }^{10}$ \\ ${ }^{1}$ Istituto Auxologico Italiano IRCCS, Department of Cardiovascular, Neurological and Metabolic Sciences, S. Luca \\ Hospital, Milan; ${ }^{2}$ Cardiology Rehabilitation Unit, ASST Rhodense, Milan; ${ }^{3}$ Cardiac Rehabilitation Unit, Istituti Clinici \\ Scientifici Maugeri IRCCS, Pavia; ${ }^{4}$ Department of Cardiology, Istituti Clinici Scientifici Maugeri IRCCS, Montescano; \\ ${ }^{5}$ Unit of Internal Medicine, Rare Disease Center, Fondazione IRCCS Ca' Granda Ospedale Maggiore Policlinico, \\ Milan and University of Milan; ${ }^{6}$ Cardiac Rehabilitation Unit, Humanitas Gavazzeni, Bergamo; ${ }^{7}$ Department of \\ Polispecialistic Anesthesia and Intensive Care, IRCCS Policlinico San Donato, S. Donato Milanese; ${ }^{8}$ Cardiac \\ Rehabilitation Unit, IRCCS Policlinico S. Donato, S. Donato Milanese; ${ }^{9}$ Cardiology Division, ASST Franciacorta, \\ Chiari; ${ }^{10}$ Department of Cardiology, Spedali Civili and University of Brescia, Italy
}

\begin{abstract}
Anemia is one of the most frequent comorbidities found in patients with coronary artery disease and chronic heart failure (CHF) who are being followed in cardiac rehabilitation facilities. The more frequent type of anemia is that caused by iron deficiency (IDA, iron-deficiency anemia): this review summarizes the state of the art of this topic. First of all, the mechanisms of IDA will be analyzed. Subsequently, a description of the main conditions where IDA can unfavorably affect the clinical course, and of its more frequent complications, will be presented (percutaneous interventions, heart surgery, CHF). Special attention will be paid in the description of anemia in the setting of CHF. To this regard, in recent years a relevant amount of research has been carried out, to determine whether treating anemia (either by directly stimulating erythropoiesis or by correcting iron deficiency by oral or intravenous route) is of any clinical and prognostic relevance in patients with CHF. The results of this research will therefore be summarized and critically discussed. Finally, we will outline the
\end{abstract}

Correspondence: Gabriella Malfatto, Istituto Auxologico Italiano IRCCS, Dipartimento di Scienze Cardiovascolari, Neurologiche, Metaboliche, Ospedale S. Luca, Piazzale Brescia 20, 20149 Milano, Italy. E-mailmalfi@auxologico.it

Key words: Cardiac rehabilitation; anemia; iron deficiency; ischemic heart disease; heart failure.

Received for publication: 25 June 2019.

Accepted for publication: 14 August 2019.

${ }^{\circ}$ Copyright: the Author(s), 2019

Licensee PAGEPress, Italy

Monaldi Archives for Chest Disease 2019; 89:1118

doi: 10.4081/monaldi.2019.1118

This article is distributed under the terms of the Creative Commons Attribution Noncommercial License (by-nc 4.0) which permits any noncommercial use, distribution, and reproduction in any medium, provided the original author(s) and source are credited. promising role of cardiac rehabilitation facilities and of its network of experts in the diagnosis, prognostic stratification and treatment of anemia and iron deficiency.

\section{Introduction}

Cardiac rehabilitation is a multidisciplinary intervention aimed at improving the outcome in terms of both the quality of life and prognosis of patients suffering a broad range of cardiovascular disease including coronary heart disease, heart failure and valvular heart disease. The comprehensive approach of cardiac rehabilitation that, apart from exercise, include clinical stabilization, optimization of pharmacological and non-pharmacological treatment, management of comorbidities and treatment of disability, is increasingly being appreciated as a successful to manage the complexities of the rapidly growing population of elderly people with cardiovascular disease [1]. Iron deficiency (ID) and iron-deficiency anemia (IDA) are highly prevalent among patients entering cardiac rehabilitation programs and are associated with health implications (including physical decline, cognitive impairment, increased susceptibility to falling) that may add to the impairment of the underlying cardiovascular disease or may limit the use of advanced therapies, as in the examples listed below. The anemiaassociated fatigue and limitation in physical performance in the post-cardiac surgery patient may not only prolong recovery time but also negatively impact on quality of life. In coronary artery disease patients submitted to percutaneous coronary intervention, anemia is a frequent cause of physician-recommended discontinuation of dual antiplatelet therapy and is associated with an increased risk of major cardiovascular and bleeding events. In the context of heart failure, anemia and ID independent of hemoglobin level, besides affecting exercise capacity and symptoms, also foster the progression of heart failure and a poor outcome. The mechanisms underlying the detrimental effects of anemia and ID in heart failure are multifactorial and not fully elucidated. Several data underscore the role of a further elevation in sympathetic activity due to the anemia-related tonic activation of excitatory chemoreceptor afferents [2]. Recent experimental studies point to the effects of ID on mitochondrial function. In a mouse model of ID without anemia, ID resulted in decreased left ventricular func- 
tion and a decrease in mitochondrial metabolism [3]. In stem-cell derived cardiomyocytes, ID was found to impair contractility and relaxation through compromised mitochondrial respiration [4]. The past twenty years have witnessed a great deal of research in the field of the pathophysiology of iron homeostasis, and the development of new iron formulations showing a low rate of immediate side effects allow to widen the field of application of iron replacement therapy. Novel diagnostic laboratory analyses and practical recommendations for the definition, diagnosis and treatment of ID have been proposed in expert consensus documents [5]. However, in spite of these recommendations, ID remains often overlooked and undertreated. Cardiac rehabilitation facilities are in a privileged position to intercept a large number of patients who may benefit from iron replacement therapy.

This paper recalls some basic concepts of iron metabolism and iron-deficiency anemia, summarizes the basic principles of treatment, highlights the size of the problem in the various patients' populations referred to cardiac rehabilitation, describes the effects of treatment and discusses how the modern cardiac rehabilitation should integrate with the treatment of this comorbidity.

\section{Iron metabolism and iron-deficiency anemia: a definition}

Anemia is a major and global public health problem, affecting more than 2 billion people and involving all age groups [6]. It is defined as a hemoglobin $(\mathrm{Hb})$ level $<12 \mathrm{~g} / \mathrm{dl}$ in women and $<13 \mathrm{~g} / \mathrm{dl}$ in men, and according to $\mathrm{Hb}$ levels it is labelled as moderate when $\mathrm{Hb}$ is between $7.0 \mathrm{~g} / \mathrm{dl}$ and $9.0 \mathrm{~g} / \mathrm{dl}$ in women and between 8.0 and $10 \mathrm{~g} / \mathrm{dl}$ in men, and severe when $\mathrm{Hb}$ is $<7.0 \mathrm{~g} / \mathrm{dl}$ in women and $<8$ $\mathrm{g} / \mathrm{dl}$ in men [6].

Epidemiology of anemia varies according to sex, age and socioeconomic status and is often multifactorial. In elderly patients one third of cases is due to nutritional deficiency, including iron, folate and vitamin $\mathrm{B}_{12}$ deficiency. Anemia of chronic disease (ACD) accounts for about another third of cases. However, in a proportion of patients, anemia cannot be explained by an underlying disease or by a specific pathological process, and for this reason almost one third of anemia in the elderly is defined "unexplained anemia”.

Half cases of worldwide anemia are due to ID, defined as "a health-related condition in which iron availability is insufficient to meet the body's needs and which can be present with or without anemia" [7]. Iron is an essential component of $\mathrm{Hb}$ in red blood cells and of myoglobin in muscles and is required for the correct function of pivotal cellular mechanisms including enzymatic processes, DNA synthesis and mitochondrial function [8]. Recently, the role of ID itself is emerging as a clinical condition irrespectively of anemia: ID per se can induce fatigue [9], alteration of cognitive performance and memory [10-12], reduction in skeletal muscle function and exercise capacity [10], increased risk of restless leg syndrome [13] and overall impaired quality of life (QoL) [12]. Adult body contains approximately 4 to 5 grams of iron: nearly 2 grams are used for red cells synthesis, $300 \mathrm{mg}$ are used daily in the bone marrow, and approximately 1 gram is stored in the liver. In a healthy subject the absorption of 1-2 mg per die balances physiological losses [5,7]. Two forms of ID are outlined below, absolute ID and functional ID.

Absolute ID occurs when total iron stores are low or depleted. It is defined by serum ferritin $<30 \mathrm{ng} / \mathrm{ml}$ and transferrin saturation (TSAT) $<20 \%$. [5]. In chronic diseases a cut-off of ferritin $<100$ $\mathrm{ng} / \mathrm{ml}$ is accepted. [5] Either physiological or pathological conditions can cause absolute ID. In infants and young children, due to the high request of iron for growth, dietary iron can be insufficient and its storage rapidly depleted. Adolescent girls and women of childbearing age are at high risk of iron deficiency because of menstrual losses; during pregnancy iron need is increased due to the expansion of the maternal red cells and growth of placenta [6].

Functional ID is defined by serum ferritin between 100 and 300 $\mathrm{ng} / \mathrm{ml}$ and TSAT $<20 \%$ [6]: in this case, iron body stores are normal or increased, but iron supply to the bone marrow is inadequate often because of chronic inflammation. Iron homeostasis is controlled by hepcidin through the internalization of ferroportin, the major iron external transporter that is expressed on enterocytes, hepatocytes and macrophages surfaces [15]. When iron level is high, hepcidin is highly expressed and ferroportin is internalized; thus, iron is trapped inside enterocytes and lost with their desquamation $[15,16]$.

During iron deficiency, hepcidin levels are low, iron can enter in the blood flow through ferroportin and can be used to form $\mathrm{Hb}$ and other molecules [5,17]. Hepcidin production and release are increased during inflammation by the activity of circulating cytokines, especially interleukin $\square 6$. This results in increased internalization and degradation of ferroportin and subsequent cellular iron retention, ultimately leading to decreased levels of circulating iron and insufficient iron availability [15-17].

\section{Basic principles of treatment of ID and IDA}

The treatment of ID and IDA is based on the identification and control of the cause and on iron supplementation. Iron can be administered orally, as a ferrous salt (i.e. ferrous sulfate, iron gluconate) or intravenously (i.v.) [18]. The choice between oral and intravenous iron is based on $\mathrm{Hb}$ levels, the expected time of correction of anemia, tolerance to oral iron supplementation and the presence of concomitant diseases that affect iron absorption: as discussed below, chronic heart failure is one of such diseases. Intravenously administration is definitely more effective to correct IDA. Although its cost is higher than oral supplementation, it offers several advantages such as rapid repletion of iron stores and reduction in hospital visits. The ideal iron i.v. product allows the administration of high amount of iron with low immunogenic risk. However, in the past, the use of iv iron was limited, due to the availability of products able to administer high quantity of iron through highly immunogenic molecules or, conversely, low immunogenic molecules able to deliver smaller amount of iron. In the last years, new products with low immunogenic risk and the ability to administer a high dose of iron in a single dose became available overcoming an unmet clinical need [17]. Ferric carboxymaltose (FCM) is a new iron product that can be administered in high single doses (up to $1000 \mathrm{mg}$ iron) with rapid infusion (15 min). FCM offers a selective delivery to bone marrow and has a low immunogenic potential being dextran-free [18]. FCM has proven safe and rapidly effective in the treatment of IDA in several settings: heavy uterine bleeding [19], inflammatory bowel disease [20], chronic kidney disease [21], perioperative anemia [22]. Treatment of ID with FCM has been recommended by the European Society of Cardiology (ESC) Guidelines for the diagnosis and treatment of acute and chronic HF (class IIa, level of evidence A), even in absence of anemia [23], as discussed below. Despite these treatment options and the advice Choosing Wisely Campaign: don't transfuse red blood cells (RBCs) for IDA without hemodynamic instability of the American Association Blood Banks 
(AABB) [24], patients with chronic IDA are still frequently transfused. Some additional considerations are warranted. First, notwithstanding AABB guidelines recommendations against transfusions in hospitalized hemodynamically stable adults including critically ill and cardiac surgery patients, RBCs transfusion remains a frequent hospital procedure at high risk of inappropriateness [25]. Second, despite the reduction of transfusion-related infection due to specific tests for known agents, exposure to transfusion carries several other risks, including transfusion-related circulatory overload and acute lung injury, allergic reaction, alloimmunization, immune suppression. Last but not least, transfusion has a high economic and social cost (Box 1).

\section{Relevance and management of ID and anemia in cardiac patients in different settings}

\section{Acute coronary syndromes and percutaneous coronary interventions}

In patients with acute coronary syndromes (ACS) prevalence of anemia ranges between 10 and $32 \%$; in many trials, anemia is an independent risk factor for increased long term mortality and major cardiac events [26]. Anemic patients undergoing percutaneous coronary interventions (PCI) are at higher risk for both ischemic and bleeding events, with a need of change in dosage, timing and duration of antithrombotic therapy [27]. When present, anemia decreases $\mathrm{O}_{2}$ delivery and the consequent increase in cardiac output, leading to chronic tachycardia, exercise myocardial ischemia and jeopardizing myocardial vitality, thus favoring adverse remodeling [28]. In anemic patients, more vulnerable plaques are observed, characterized by higher burden of necrotic core and thin cap fibroatheroma. If anemia is caused by bleeding, dual antiplatelet therapy has to be discontinued prematurely and this can lead to stent thrombosis and obviously worse outcome. [29]. Of note, in ACS anemia is also a marker of comorbidities as age, diabetes, chronic kidney failure and autoimmune diseases. In patients suffering from ACS, perhaps aggravated by an accompanying contractile impairment, guidelines are more focused on priorities such as the need for pharmacological and mechanical support [30] and the interventional approach, so that the management of anemia is poorly defined: the management of the anemic patient is based on optimization and stabilization of the circulation through the use of transfusions (with a liberal level of hemoglobin $<9.0 \mathrm{~g} / \mathrm{dl}$ ) without standardized modalities. Indeed, a recent study showed that ID in the setting of ACS is strongly associated with adverse outcome [31]; therefore, a treatment addressed to solve any ID condition would sustain energy mechanisms and limit the risk of post-procedural anemia and the consequent need for transfusions.

\section{Box 1 - Why transfusions are to be avoided?}

\author{
RBCs transfun are at high risk of inappropriateness \\ Exposure to transfusion carries risk of \\ - transfusion-related circulatory overload and acute lung injury; \\ - allergic reaction; \\ - alloimmunization; \\ - immune suppression. \\ Transfusion has a high economic and social cost
}

\section{Cardiac surgery}

Anemia is a frequent condition in cardiac surgery, its overall prevalence being $25-30 \%$ [32,33] with a slight prevalence in males [32]. Since the most important cause of surgical anemia is hospitalinduced anemia [33], in most patients the preoperative anemia is preventable. Patients with preoperative anemia have reduced red blood indices (i.e. mean corpuscular hemoglobin and/or mean corpuscular volume); patients with reduced cell indices are younger, show lower perioperative hemoglobin and have higher transfusion rates compared to those with normal red cell indices [33]. As in non-cardiac surgery, in cardiac surgery anemia is linked to surgical mortality and morbidity; however, the type of surgery (cardiac vs non-cardiac) and the presence of cardiovascular disease in patients undergoing general surgery carry an increased mortality risk [33]. Since anemia is often associated with other comorbid conditions, as chronic kidney disease, older age and poor left ventricular function, its role as an independent risk factor has not been established [3135]: as a consequence, preoperative anemia is not included in risk scores such as Euroscore [36] but hematocrit is present in STS [37]. Anemia leads to higher transfusion rates; in some reports, even the transfusion of a single RBC unit led to increased morbidity and mortality, the risk increasing with transfusion of more units [38]; in others, transfusion of 1-2 units of red blood cells did not increase mortality but was associated with an increased risk of acute kidney insufficiency, sternal wound infection, stroke, prolonged ICU and in-hospital stay and prolonged mechanical ventilatory support [39]. There is agreement on the concept that perioperative red blood cell transfusion of more than two units represents the single factor most reliably associated with increased risk of morbid events after coronary artery bypass grafting [40]. Engoren et al. [41] discriminated whether anemia, transfusion or the inflammatory state linked to these two conditions was the real dangerous factor, considering 4 groups of patients: anemic and transfused patients had the highest death hazard, patients who were not anemic and had not been transfused had the lowest hazard, patients anemic but not transfused and patients transfused but non-anemic had intermediate death hazard. Prognosis worsened when major bleeding or preoperative chronic kidney disease were associated with anemia and/or transfusion. [42]. Thus, preoperative correction of iron deficiency by intravenous iron might be beneficial through the correction of ID per se or through the correction of anemia [43]. In conclusion, anemia is not an innocent bystander, on the contrary it represents a contraindication to elective procedures and should be managed before their performance, as detailed below [44].

The invasiveness and traumatic effects of surgical interventions, through a cascade of inflammatory responses $[45,46]$ influence the volume of blood loss [47] that may in turn be amplified by the overlap of coagulopathies that further promote bleeding [48]. A typical example is cardiac surgery, where extracorporeal circulation (ECC) modifies the coagulation function. ECC requires a reversible, pharmacologically-induced anticoagulation state [49]. However, several factors such as residual heparin effect, reduced thrombin generation, fibrinogen deficiency, thrombocytopenia, platelet dysfunction and hyperfibrinolysis could be present in the postoperative phase and cause a propensity to bleeding [50], whose entity will determine the extent of patient's anemic response [50]. The reduced hemoglobin concentration in healthy patient is compensated by an increase in cardiac output to ensure adequate tissue $\mathrm{O}_{2}$ delivery [52,53]. In patients with cardiac disease, this compensatory function is often inadequate [54] leaving them vulnerable to low levels of circulating hemoglobin $(\mathrm{Hb})$. Thus, even if international guidelines recommend restrictive transfusion criteria $(\mathrm{Hb} 7.0 \mathrm{~g} / \mathrm{dl})$, they acknowledge the 
fragility of cardiac patients and consent to transfuse them at higher hemoglobin levels ( $\mathrm{Hb} 8 \mathrm{~g} / \mathrm{dl})$ [55-58]. The high risk of bleeding and anemia increase the risk of postoperative transfusion in patient undergoing cardiac surgery. Preoperative anemia is a modifiable risk factor [59] for increased postoperative transfusion [60], so that its correction can reduce the incidence of negative outcomes [61]. Following WHO anemia definition [62], anemia affects about $27 \%$ of the world's population. A slightly higher prevalence is found in patients scheduled for cardiac surgery $(\approx 30 \%)$, in whom we may expect an additional blood loss in the perioperative period [63]. Moreover, women are subject to greater blood loss and more transfusions than men undergoing similar procedures [64]. Thus, from the surgeon's point of view a hemoglobin level $<13 \mathrm{~g} / \mathrm{dl}$ is inadequate for both sexes, and $40 \%$ the cardiac surgery population would have suboptimal $\mathrm{Hb}$ values that should be treated in a surgical preparation program. Such aggressive approach has been suggested by a recent international consensus document regarding Patient Blood Management (PBM) [65]. The most frequent anemia found in patients scheduled for cardiac surgery is ID anemia [66,67], followed by anemia accompanying kidney disease [68,69]. Around three to four weeks before surgery, an appropriate screening of the iron status helps to recognize anemia and look for its etiology, allowing adequate treatment [65]. The increase in preoperative hemoglobin would minimize the loss expected in the postoperative period $[68,69]$, lessen the two nadirs of hemoglobin (during ECC and in intensive care), prevent the need for transfusions [70,71].

Remarkably, when anemia is observed after surgery in a postsurgical rehabilitation setting, it does not contraindicate or hinder rehabilitation programs. In a study by Ranucci et al. [26], hemoglobin levels of 8 to $10 \mathrm{~g} / \mathrm{dl}$ at the admittance in Rehabilitation were associated to a transient impairment of exercise tolerance (measured by 6-min walking test) directly proportional to the severity of anemia; this functional gap completely recovered during the rehabilitation period. Thus, anemia does not contraindicate rehabilitation even if anemic patients have a slow recovery in their physical performance with respect to non-anemic patients; in fact, the same Authors showed that blood transfusion in the rehabilitation setting did not increase exercise tolerance at the 6-min walking test [72]. These conclusions have been confirmed by a recent study, where the Authors showed a high prevalence of ID and a $10 \%$ ID anemia in the setting of post-surgical rehabilitation, and suggested that the rehabilitation phase may be suitable for screening and correction of ID and ID anemia [73].

\section{Heart failure}

Anemia and ID often coexist in patients with chronic heart failure $(\mathrm{CHF})$. Both conditions, together or independently, are associated with poor clinical status and worse outcome.

Knowing anemia etiology is fundamental to treat it in the best possible way. As detailed above, iron deficiency and inflammation play a pivotal role, but heart failure anemia may have additional causes:

i) reduced intestinal iron absorption due to abnormal hepcidin and ferroportin activity in gut cells;

ii) increased cytokine levels, causing bone marrow blood cells underproduction;

iii) activation of renin-angiotensin-aldosterone system, determining hemodilution through sodium and water retention;

iv) reduced erythropoietin (EPO) production due to kidney failure and at least in part to angiotensin converting enzyme inhibitors treatment;

v) the use of antiplatelet or anticoagulant agents and the consequent risk of bleeding.
Whether anemia and ID are just markers of HF severity or whether they mediate HF progression and outcomes is not entirely clear $[74,75]$. As stated above, ID in patients with HF can be absolute or functional, and even in the absence of anemia, absolute or functional ID represents a major contributor to exercise intolerance and an independent predictor of outcome [75,76]. Although ID is highly prevalent in patients with CHF, this condition is often neglected because symptoms related to ID and those related to $\mathrm{CHF}$ (fatigue and weakness, dyspnea) may overlap: only the assessment of plasmatic iron parameters allows ID diagnosis. Regardless of its causes, high prevalence of ID in CHF has been reported in many cohorts of patients, ranging from $37 \%$ (32\% in non-anemic patients) [75] and 50\% (46\% in non-anemic patients) [76,77]. The current European Society of Cardiology guidelines [23] suggest a periodical assessment of ferritin, transferrin, iron plasma levels and transferrin saturation (TSAT) in CHF patients, together with the search for the cause of ID and anemia. Treatment for ID involves the restoration of iron stores to normal levels and the increase of hemoglobin values, as well as addressing its underlying causes [23].

Anemia and ID status have different therapeutic possibilities: blood transfusions in acute and hemodynamically unstable patients, erythropoiesis-stimulating agents and iron replacement, either oral or intravenous, in chronic patients. The use of erythropoiesis-stimulating agents, albeit initially promising [78], has been abandoned and is currently not recommended [23]. Iron replacement is the treatment of choice. However, oral iron preparations do not seem effective in patients with HF with reduced ejection fraction (HFrEF). In the IRONOUT-HF trial, oral iron polysaccharide supplementation did not improve exercise capacity or quality of life scores, as assessed by Kansas City Cardiomyopathy Questionnaire (KCCQ) [79].

Among the several intravenous iron preparations available in Europe, FCM has been largely experienced in HF in two large trials: the "Ferinject Assessment in Patients with Iron Deficiency and Chronic Heart Failure (FAIR-HF)" (n=459) [80] and "A Study to Compare the Use of Ferric Carboxymaltose With Placebo in Patients With Chronic Heart Failure and Iron Deficiency (CONFIRM-HF)" $(n=301)$ [81]. The FAIR-HF study was as a multicenter, prospective, double-blind, randomized, placebo-controlled trial [80]. Symptoms, functional capacity and QoL were significantly improved after treatment with IV FCM in patients with chronic HF and ID, with or without anemia. The CONFIRM-HF trial [81] included 301 patients with moderate HF (NYHA Class II-III), left ventricular EF (LVEF) $\leq 45 \%$, increased levels of BNP $>100 \mathrm{pg} / \mathrm{mL}$ and/or NT-proBNP $>400 \mathrm{pg} / \mathrm{mL}$, and ID. IV iron was given as an FCM solution equivalent to 500 or $1,000 \mathrm{mg}$ of iron. At week 24 , the 6MWT distance improved significantly more in the FCM group; the benefit was maintained up to 52 weeks. Fatigue and QoL scores also improved significantly up to week 52, as well as NYHA class and patient global assessment scores. Furthermore, FCM was associated with a significant reduction in the risk of hospitalization due to worsening HF. Of note, in a sub analysis of the FAIR-HF trial by Ponikowski et al. [82], FCM treatment was associated with an improvement in renal function across the entire spectrum of HF patients enrolled in the trial. This result was present also in the CONFIRM-HF trial [81]. Finally, the recent EFFECT-HF trial (Effect of Ferric Carboxymaltose on Exercise Capacity in Patients with Iron Deficiency and Chronic Heart Failure) [83] demonstrated an improvement in $\mathrm{VO}_{2}$ max in 172 symptomatic HFrEF patients treated with FCM in comparison with the placebo group.

In addition, meta-analyses confirmed these results [84,85]: for instance, IV iron therapy in ID patients with systolic HF induced a significant reduction in the combined endpoints of all-cause death 
or cardiovascular hospitalizations, cardiovascular death or hospitalization for worsening HF; moreover, a significant reduction in NYHA class, an increase in the six-minute walking test (6MWT) distance, and an improvement in QoL were reported [84]. Following these evidences, the $2016 \mathrm{HF}$ ESC guidelines [77] and the 2017 ACC/AHA/HFSA update of the 2013 ACCF/AHA guidelines for the management of heart failure [86] recommend treatment with FCM in HF patients with iron deficiency who remain symptomatic despite optimal therapy, in order to alleviate symptoms, improve exercise capacity and quality of life (classes of recommendations and level of evidence: IIa A and IIb B, respectively, in European and American guidelines).

So far, it is unclear whether iron therapy affects patients with midrange and preserved left ventricular ejection fraction, since most trials excluded patients with left ventricular ejection fraction greater than $45 \%$ [87]. The ongoing FAIR-HfpEF trial (Effect of IV Iron in Patients with Heart Failure with Preserved Ejection Fraction; NCT03074591) will assess whether FCM treatment can improve exercise capacity, by changes in 6MWT distance, in HF patients with left ventricular ejection fraction above than $45 \%$. Another ongoing trial will investigate the gray area of acute heart failure: the AFFIRM-AHF (Study to Compare Ferric Carboxymaltose with Placebo in Patients with Acute Heart Failure and Iron Deficiency; NCT02937454) will randomize patients with left ventricular ejection fraction $<50 \%$ after acute care treatment. Furthermore, other trials will increase the information in patients with HFrEF, such as the FAIR-HF2 (Intravenous Iron in Patients with Systolic Heart Failure and Iron Deficiency to Improve
Morbidity and Mortality; NCT03036462) or IRON-MAN (Intravenous Iron Treatment in Patients with Heart Failure and Iron Deficiency; NCT 02642562). Figure 1 illustrates the studies currently ongoing on IV iron treatment for ID and IDA in various forms of heart failure.

Most CHF patients are not hospitalized for cardiac rehabilitation but are followed in outpatients' specialized HF units. How can we organize the quest for ID and ID anemia in this setting? Realworld data on the efficacy of intravenous FCM therapeutics in current clinical practice are still scarce [88-90]. Clinical trials have typically evaluated patients aged 65.70 years, excluding those with co-morbidities. To this regard, a single-center double-blind randomized pilot study on 70 patients from a rehabilitation center in Milano showed the feasibility and reliability of IV iron replacement with FCM [91]. Anemia and ID were defined with usual criteria [77]. Patients underwent symptoms evaluation, blood tests and cardiopulmonary exercise test before, one month and three months after IV iron therapy, targeted to replenish iron stores; NYHA class and peakVO2 at cardiopulmonary test improved only in the active arm group (Figure 2). From this experience, one may conclude that treatment of iron deficient anemia is feasible and easy to organize in an outpatients' setting, and likely to afford a significant clinical benefit to chronic heart failure patients.

However, we must take care in not to over-treat patients, since this may determine an increase of oxidative stress, increasing levels of malondialdheyde and asymmetric dimethylarginine, a biomarker of lipid peroxidation and one of the most important agents causing endothelial dysfunction, respectively $[92,93]$.

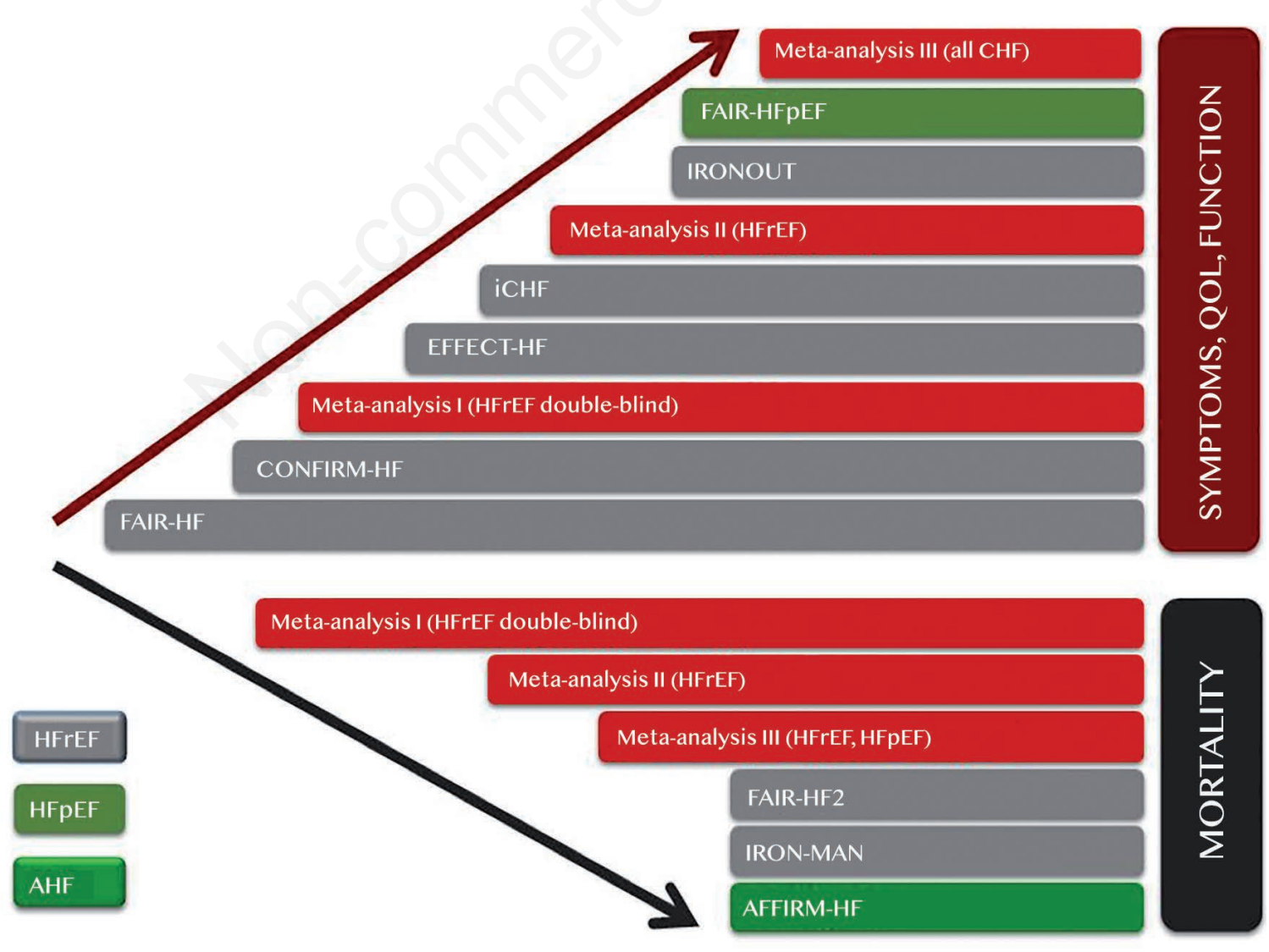

Figure 1. An overview of the studied researches on ID and IDA. 


\section{Key role of cardiac rehabilitation in the diagnosis and treatment of ID and IDA}

Anemia and iron deficiency may potentially affect all patients during cardiac rehabilitation (CR) programs, regardless of primary condition for referral, with high prevalence rates and strong association to disease severity, as far as to worse outcomes [94,95]. Anemia and ID also contribute to impaired functional capacity [87] and increased disability [96]. It seems therefore reasonable to search for and treat ID and IDA, especially in the setting of phase II CR activities.

During the past recent years, anemia and IDA have emerged as highly frequent comorbidities in several subsets of patients entering phase II cardiac rehabilitation. Optimization of therapy (and accordingly the diagnostic work-up preceding the treatment) is one of the core components of a modern and multidisciplinary cardiac rehabilitation. Thus, we believe that phase II cardiac rehabilitation that usually lasts from three to six weeks and, in addition to physical training, involves continuous monitoring of patients' clinical status and treatment optimization, may in fact offer a valuable opportunity to comprehensively manage all those clinical conditions that can negatively impact recovery, quality of life and eventually physical functioning. Such approach would be appropriate especially in patients with chronic heart failure (CHF), where ID and IDA have been extensively investigated, leading to the current recommendations of the 2016 ESC guidelines [77].

Indeed, a modern $\mathrm{CR}$ program - where modern indicates the care of highly complex and frail patients - would be expected to consider ID and ID anemia as important determinants of the rehabilitative outcome, in terms of i) clinical stabilization; ii) reduction of exercise intolerance; and iii) promotion of better quality of life. Noticeably, such an approach should also take into account the different modalities of presentation of patients (e.g. patients with recent hospitalization in acute wards due to hemodynamic instability, or patients in the "vulnerable phase" after index events, or patients in the chronic phase) and the different CR settings (residential, out-patient, or home-rehabilitation).

The definition of symptomatic patient should also be interpreted in a holistic way - rather than just a grading of dyspnea and derived NYHA class - in order to integrate detailed information on functional capacity and fatigue: many of these direct and indirect evaluations are routinely performed during $\mathrm{CR}$ activities and could help identifying appropriate candidates for the intensive correction of ID and ID anemia. Indeed, intravenous FCM, in appropriately selected subjects and at the appropriate time, may boost the efficacy of physical training, due to its documented effects on muscle metabolism; in this perspective, the CR setting could really demonstrate the efficacy of such a composite therapeutic strategy [73,91] (Box 2). Nonetheless, several features of this strategy are still controversial, so that we do not have so far a clear indication for routine practice.

First, we should identify the proper timing for ID evaluation during the CR program; as an example, is it appropriate in patients fast-tracked from acute wards, often presenting with persistent symptoms of fluid retention?

Second, we need to organize and implement a proper followup of patients actively treated for ID and IDA: to this regard, ESC guidelines do not provide adequate recommendations.

Moreover, how do we define partially and no-responder patients? Which amount of increase in exercise tolerance clinicians could reasonably expect by correcting ID and IDA, and how to define positive and negative outcomes?

Finally, there is strong need of a detailed cost-effectiveness analysis, specifically tailored on CR activities with special regard on the residential setting.
NYHA III-IV, \%

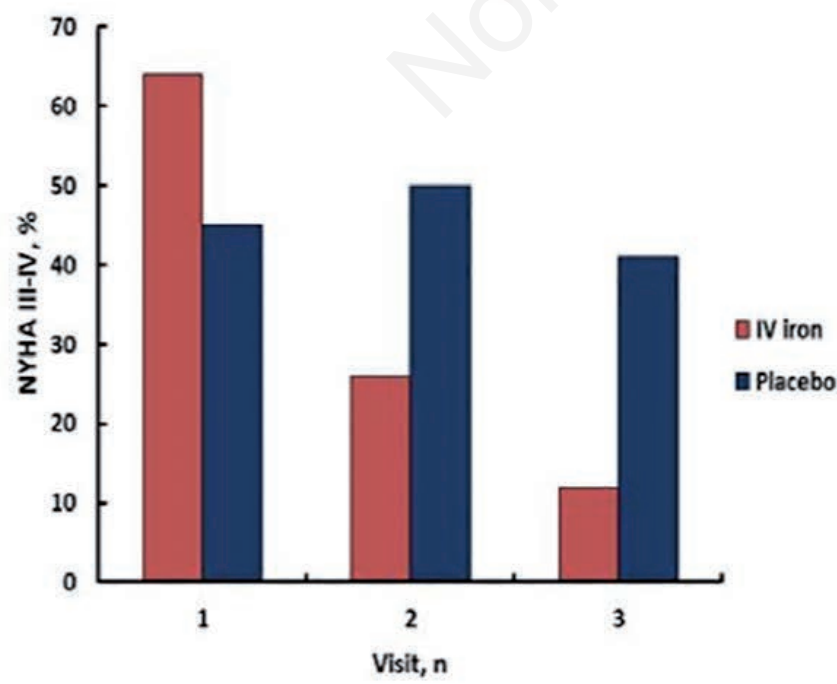

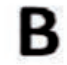

B Peak oxygen consumption

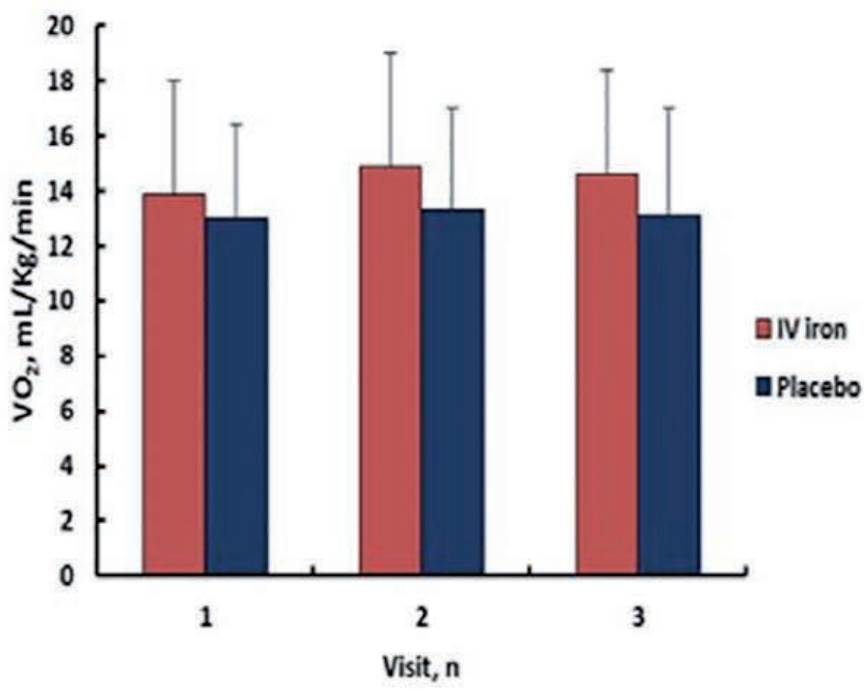

Figure 2. A) NYHA class improved in the active arm group from baseline (visit 1) to visits 2 and 3. B) PeakVO at cardiopulmonary test slightly improved (about $1.5 \mathrm{~mL} / \mathrm{Kg} / \mathrm{min}$ ) in the active arm group and remained unchanged in the control group. 


\section{Box 2 - Proposal for an outpatients' management of ID and IDA.}

\section{Search periodically for ESC guidelines criteria for IV iron replacement (the iron is the target!)}

- When? Every year

- Who? HFrEF patients

- What? serum ferritin $<100 \mu \mathrm{g} / \mathrm{L}$, or ferritin 100-299 $\mu \mathrm{g} / \mathrm{L}$ and transferrin saturation $<20 \%$

\section{Administer i.v. FCM in an outpatients' basis}

- How? 1-3 morning sessions with ECG and peripheral $\mathrm{O}_{2}$ monitoring.

- Who is in charge? A nurse must be present, a referring cardiologist should be available on call

\section{References}

1. Pedretti RFE, Fattirolli F, Griffo R, et al. Cardiac Prevention and Rehabilitation "3.0": From acute to chronic phase. Position Paper of the ltalian Association for Cardiovascular Prevention and Rehabilitation (GICR-IACPR). Monaldi Arch Chest Dis 2018;88:1004. Doi: 10.4081/monaldi.2018.1004

2. Franchitto N, Despas F, Labrunée M, et al. Tonic chemoreflex activation contributes to increased sympathetic nerve activity in heart failure-related anemia. Hypertension 2010;55:1012-7.

3. Rineau E, Gaillard T, Gueguen N, et al. Iron deficiency without anemia is responsible for decreased left ventricular function and reduced mitochondrial complex I activity in a mouse model. Int J Cardiol 2018;266:206-12.

4. Hoes MF, Grote Beverborg N, Kijlstra JD, et al. Iron deficiency impairs contractility of human cardiomyocytes through decreased mitochondrial function. Eur J Heart Fail 2018; 20:910-9.

5. Cappellini MD, Comin-Colet J, de Francisco A, et al. IRON CORE Group. Iron deficiency across chronic inflammatory conditions: International expert opinion on definition, diagnosis, and management. Am J Hematol 2017;92:1068-78.

6. Kassebaum NJ, Jasrasaria R, Naghavi M, et al. A systematic analysis of global anemia burden from 1990 to 2010. Blood 2014;123:615-24.

7. Cappellini MD, Motta I. Anemia in clinical practice, definition and classification: Does Hemoglobin change with aging? Semin Hematol 2015;52:261-9.

8. Andrews NC. Forging a field: the golden age of iron biology. Blood 2008;112:219-30.

9. Verdon F, Burnand B, Stubi CL, et al. Iron supplementation for unexplained fatigue in non-anaemic women: double blind randomised placebo controlled trial. BMJ 2003;326:1124-9.

10. Congdon EL, Westerlund A, Algarin CR, et al. Iron deficiency in infancy is associated with altered neural correlates of recognition memory at 10 years. J Pediatr 2012;160:1027-33.

11. Beard JL, Hendricks MK, Perez EM, et al. Maternal iron deficiency anemia affects postpartum emotions and cognition. J Nutr 2005; 135:267-72.

12. Andro M, Le Squere P, Estivin S, Gentric A. Anaemia and cognitive performances in the elderly: a systematic review. Eur J Neurol 2013;20:1234-40.

13. Trenkwalder C, Allen R, Hogl B, et al. Restless leg syndrome associated with major diseases: a systematic review and new concept. Neurology 2016;86:1336-43.

14. De Franceschi L, Iolascon A, Taher A, Cappellini MD. Clinical management of iron deficiency anemia in adults: Systemic review on advances in diagnosis and treatment. Eur $\mathrm{J}$ Intern Med 2017;42:16-23.

15. Ganz T. Hepcidin, a key regulator of iron metabolism and mediator of anemia of inflammation. Blood 2003;102:783-88.

16. Ganz T. Systemic iron homeostasis. Physiol Rev 2013;93: 1721-41.

17. Camaschella C. Iron deficiency: new insights into diagnosis and treatment. Hematology Am Soc Hematol Educ Program 2015;2015:8-13.

18. Girelli D, Ugolini S, Busti F, et al. Modern iron replacement therapy: clinical and pathophysiological insights. Int J Hematol 2018;107:16-30.

19. Van Wyck DB, Mangione A, Morrison J et al. Large-dose intravenous ferric carboxymaltose injection for iron deficiency anemia in heavy uterine bleeding: a randomized, controlled trial. Transfusion 2009;49:2719-28.

20. Evstatiev R, Marteau P, Iqbal T, et al. FERGIcor, a randomized controlled trial on ferric carboxymaltose for iron deficiency anemia in inflammatory bowel disease. Gastroenterology 2011;141:846-53.

21. Macdougall IC, Bock AH, Carrera F, et al. FIND-CKD: a randomized trial of intravenous ferric carboxymaltose versus oral iron in patients with chronic kidney disease and iron deficiency anemia. Nephrol Dial Transplant 2014;29:2075-84.

22. Kim YW, Bae JM, Park YK, et al. Effect of intravenous ferric carboxymaltose on hemoglobin response among patients with acute isovolemic anemia following gastrectomy. JAMA 2017;317:2097-104.

23. Ponikowski P, Voors AA, Anker SD, et al. 2016 ESC Guidelines for the diagnosis and treatment of acute and chronic heart failure: The Task Force for the diagnosis and treatment of acute and chronic heart failure of the European Society of Cardiology (ESC). Developed with the special contribution of the Heart Failure Association (HFA) of the ESC. Eur J Heart Fail 2016;18:891-975.

24. Callum JL, Waters JH, Shaz BH, et al. The AABB recommendations for the Choosing Wisely campaign of the American Board of Internal Medicine. Transfusion 2014;54:2344-52.

25. Muñoz M, Gómez-Ramírez S, Campos A, et al. Pre-operative anaemia: prevalence, consequences and approaches to management. Blood Transfus 2015;13:370-9.

26. Ranucci M, La Rovere MT, Castelvecchio S, et al. Postoperative anemia and exercise tolerance after cardiac operations in patients without transfusion: what hemoglobin level is acceptable? Ann Thorac Surg 2011;92:25-31.

27. Al-Hijji MA, Gulati R, Lennon RJ, et al. Outcomes of percutaneous coronary interventions in patients with anemia presenting with acute coronary syndrome. Mayo Clin Proc 2018;93:1448-61. 
28. Anderson GJ, Vulpe CD. Mammalian iron transport. Cell Mol Life Sci 2009;66:3241-61.

29. Wang X, Qiu M, Qi J, et al. Impact of anemia on long-term ischemic events and bleeding events in patients undergoing percutaneous coronary intervention: a system review and meta-analysis. J Thorac Dis 2015;7:2041-52.

30. Ibanez B, James S, Agewall S, et al. 2017 ESC Guidelines for the management of acute myocardial infarction in patients presenting with ST-segment elevation. Eur Heart J 2018;39:119-77.

31. Zeller T, Waldeyer C, Ojeda F, et al. Adverse outcome prediction of iron deficiency in patients with acute coronary syndrome. Biomolecules 2018;8:60-9.

32. Engoren M, Schwann TA, Habib RH, et al. The Independent effects of anemia and transfusion on mortality after coronary artery bypass. Ann Thorac Surg 2014;97:514-21.

33. Davis O, Sinha R, Robinson K, Cardone D. The prevalence of anaemia, hypochromia and microcytosis in preoperative cardiac surgical patients. Anaesth Intensive Care 2013;41:316-21.

34. Karski JM, Mathieu M, Cheng D, et al. Etiology of preoperative anemia in patients undergoing scheduled cardiac surgery. Can J Anesth 1999;46:979-82.

35. Ranucci M, Di Dedda U, Castelvecchio S, et al. Impact of preoperative anemia on outcome in adult cardiac surgery: a propensity-matched analysis. Ann Thorac Surg 2012;94:1134-42 .

36. Parolari A, Pesce LL, Trezzi M, et al. Performance of EuroSCORE in CABG and off-pump coronary artery bypass grafting: single institution experience and meta-analysis. Eur Heart J 2009;30:297-304.

37. Sullivan PG, Wallach JD, Ioannidis JP. Meta-analysis comparing established risk prediction models (EuroSCORE II, STS score, and ACEF score) for perioperative mortality during cardiac surgery. Am J Cardiol 2016;118:1574-82.

38. Ferraris VA, Davenport DL, Saha SP, et al. Surgical outcomes and transfusion of minimal amounts of blood in the operating room. Arch Surg 2012;147:49-55.

39. Koster A, Zittermann A, Börgermann J, et al. Transfusion of 1 and 2 units of red blood cells does not increase mortality and organ failure in patients undergoing isolated coronary artery bypass grafting. Eur J Cardiothorac Surg 2016;49:931-6.

40. Ranucci M, Baryshnikova E, Castelvecchio S, et al. Major bleeding, transfusions, and anemia: The deadly triad of cardiac surgery. Ann Thorac Surg 2013;96:478-85.

41. Engoren M, Schwann TA, Habib RH, et al. The independent effects of anemia and transfusion on mortality after coronary artery bypass. Ann Thorac Surg 2014;97:514-20.

42. Ogami T, Matsue Y, Kawasumi R, Tanabe H. Prognostic implications of preoperative chronic kidney disease and anemia in patients undergoing coronary artery bypass graft surgery. Surg Today 2017;47:245-51.

43. Piednoir P, Allou N, Driss F, et al. Preoperative iron deficiency increases transfusion requirements and fatigue in cardiac surgery patients: a prospective observational study. Eur J Anaesthesiol 2011; 28: 796-801

44. Spahn DR, Zacharowski K. Non-treatment of preoperative anaemia is substandard clinical practice. Brit $\mathrm{J}$ Anesthesiol 2015;115:1-3.

45. Ripolles-Melchor J, Carli F, Coca-Martínez Met al. Committed to be fit. The value of preoperative care in the perioperative medicine era. Minerva Anesthesiologica 2018;84:615-25.

46. Pearse RM, Harrison DA, James P et al. Managing perioperative risk in patients undergoing elective non-cardiac surgery. BMJ 2011;343:d5759.

47. Stokes ME, Ye X, Shah M et al. Impact of bleeding-related complications and/or blood product transfusions on hospital costs in inpatient surgical patients. BMC Health Serv Res 2011;11:135-40.

48. Marietta M, Facchini L, Pedrazzi P, et al. Pathophysiology of bleeding in surgery. Transplant Proc 2006;38:812-4.

49. Despotis GJ, Joist JH. Anticoagulation and anticoagulation reversal with cardiac surgery involving cardiopulmonary bypass: an update. J Cardiothorac Vasc Anesth 1999;13:s1829. discussion 36-7.

50. Ranucci M. Hemostatic and thrombotic issues in cardiac surgery. Semin Thromb Hemost 2015;41:84-90.

51. Lasocki S, Krauspe R, von Heymann C, et al. PREPARE: the prevalence of perioperative anaemia and need for patient blood management in elective orthopaedic surgery. Eur J Anaesthesiol 2015; 32:160-7.

52. Hare GMT. Tolerance of anemia: understanding the adaptive physiological mechanisms which promote survival. Transfus Apher Sci 2014;50:10-2.

53. Greenburg AG. A physiologic basis for red blood cell transfusion decisions. Am J Surg 1995; 170:44S-8.

54. Frank SM, Ejaz A, Pawlik TM. Optimal transfusion trigger in surgical patients with coronary artery disease. JAMA Surgery 2016;151:146.

55. Docherty AB, O'Donnell R, Brunskill S, et al. Effect of restrictive versus liberal transfusion strategies on outcomes in patients with cardiovascular disease in a non-cardiac surgery setting: systematic review and meta-analysis. BMJ 2016;352:i1351.

56. Carson JL, Guyatt G, Heddle NM, et al. Clinical practice guidelines from AABB - Red blood cell transfusion thresholds and storage. JAMA 2016;316:2025-35.

57. Boer C, Meesters MI, Milojevic M, et al. 2017EACTS/ EACTA Guidelines on patient blood management for adult cardiac surgery. J Cardiothorac Vasc Anesth 2018;32:88-120.

58. Kozek-Langenecker SA, Ahmed AB, Afshari A, et al. Management of severe perioperative bleeding: guidelines from the European Society of Anaesthesiology - First update 2016. Eur J Anaesthesiol 2017;34:332-95.

59. Chakravarthy M. Modifying risks to improve outcome in cardiac surgery: an anesthesiologist's perspective. Ann Card Anaesth 2017;20:226-33.

60. Klein AA, Collier TJ, Brar MS, et al. The incidence and importance of anaemia in patients undergoing cardiac surgery in the UK - the first Association of Cardiothoracic Anaesthetists national audit. Anaesthesia 2016;71:627-35.

61. Loor G, Koch CG, Sabik JF 3rd, et al. Implications and management of anemia in cardiac surgery: current state of knowledge. J Thorac Cardiovasc Surg 2012;144:538-46.

62. WHO. Haemoglobin concentrations for the diagnosis of anaemia and assessment of severity. Vitamin and Mineral Nutrition Information System. Geneva, World Health Organization, 2011. Available from: https://www.who.int/ vmnis/indicators/haemoglobin/en/

63. Muñoz M, Laso-Morales MJ, Gómez-Ramírez S, et al. Preoperative anaemia: prevalence, consequences and approaches to management. Blood Transfus 2015;13:370-9.

64. Blaudszun G, Munting KE, Butchart A, et al. The association between borderline preoperative anaemia in women and outcomes after cardiac surgery: a cohort study. Anaesthesia 2018;73:572-8.

65. Meybohm P, Fischer DP, Geisen C, et al. Safety and effectiveness of a Patient Blood Management (PBM) program in surgical patients - the study design for a multi-centre prospective 
epidemiologic non-inferiority trial. BMC Health Serv Res 2014;14:576-81.

66. Hung M, Ortmann E, Besser M, et al. A prospective observational cohort study to identify the causes of anaemia and association with outcome in cardiac surgical patients. Heart 2015;101:107-12.

67. Grey DE, Smith V, Finlayson J. Iron studies and red cell transfusion in cardiothoracic and orthopaedic surgical patients: a retrospective audit at a tertiary hospital. Vox Sanguinis 2012;102:150-8.

68. Grant MC, Whitman GJ, Savage WJ, et al. Clinical predictors of postoperative hemoglobin drift. Transfusion 2014;54:1460-68.

69. George TJ, Beaty CA, Kilic A, et al. Hemoglobin drift after cardiac surgery. Ann Thorac Surg 2012;94:703-9.

70. Scott DA, Tung HMA, Slater R, et al. Perioperative hemoglobin trajectory in adult cardiac surgical patients. J Extra Corp Technol 2015;47:167-73.

71. Hogervorst E, Rosseel P, van der Bom J, et al. Tolerance of intraoperative hemoglobin decrease during cardiac surgery. Transfusion 2014;54:2696-704.

72. Ranucci M, La Rovere MT, Castelvecchio S, et al. Effects of red blood cell transfusions on exercise tolerance and rehabilitation time after cardiac surgery. Transfus Apher Sci 2011;45:299-303.

73. Tramarin R, Pistuddi V, Maresca L, et al. Patterns and determinants of functional and absolute iron deficiency in patients undergoing cardiac rehabilitation following heart surgery. Eur J Prev Cardiol 2017;24:799-807.

74. Anand IS, Gupta P. Anemia and iron deficiency in heart failure. Current concepts and emerging therapies. Circulation 2018;138:80-98.

75. Jankowska EA, Kasztura M, Sokolski M, et al. Iron deficiency defined as depleted iron stores accompanied by unmet cellular iron requirements identifies patients at the highest risk of death after an episode of acute heart failure. Eur Heart J 2014;35:2468-76.

76. Jankowska EA, Rozentryt P, Witkowska A, et al. Iron deficiency: an ominous sign in patients with systolic chronic heart failure. Eur Heart J. 2010;15: 1872-80.

77. Klip IT, Comin-Colet J, Voors AA, et al. Iron deficiency in chronic heart failure: an international pooled analysis. Am Heart J 2013;165:575-82.

78. Silverberg DS, Wexler D, Blum M, et al. The use of subcutaneous erythropoietin and intravenous iron for the treatment of the anemia of severe, resistant congestive heart failure improves cardiac and renal function and functional cardiac class, and markedly reduces hospitalizations. J Am Coll Cardiol 2000;35:1737-44.

79. Lewis GD, Malhotra R1, Hernandez AF, et al. Effect of oral iron repletion on exercise capacity in patients with heart failure with reduced ejection fraction and iron deficiency: the IRONOUTHF Randomized Clinical Trial. JAMA 2017;317:1958-66.

80. Anker SD, Comin Colet J, Filippatos G, et al. Ferric carboxymaltose in patients with heart failure and iron deficiency. $\mathrm{N}$ Engl J Med 2009;361:2436-48.

81. Ponikowski P, van Veldhuisen DJ, Comin-Colet $J$, et al. Beneficial effects of long-term intravenous iron therapy with ferric carboxymaltose in patients with symptomatic heart failure and iron deficiency. Eur Heart J 2015;36:657-68.
82. Ponikowski P, Filippatos G, Colet JC, et al. The impact of intravenous ferric carboxymaltose on renal function: an analysis of the FAIR-HF study. Eur J Heart Fail 2015;17:329-39.

83. Van Veldhuisen DJ, Ponikowski P, van der Meer P, et al. Effect of ferric carboxymaltose on exercise capacity in patients with chronic heart failure and iron deficiency. Circulation 2017;136:1374-83.

84. Bolger AP, Bartlett FR, Penston HS, et al. Intravenous iron alone for the treatment of anemia in patients with chronic heart failure. J Am Coll Cardiol 2006;48:1225-7.

85. Jankowska EA, Tkaczyszyn M, Suchocki T, et al. Effects of intravenous iron therapy in iron-deficient patients with systolic heart failure: a meta-analysis of randomized controlled trials. Eur J Heart Fail 2016;18:786-95.

86. Yancy CW, Jessup M, Bozkurt B, et al. 2017 ACC/AHA/HFSA Focused Update of the 2013 ACCF/AHA Guideline for the Management of Heart Failure: A Report of the American College of Cardiology/American Heart Association Task Force on Clinical Practice Guidelines and the Heart Failure Society of America. J Am Coll Cardiol 2017;70:776-803.

87. Martens P, Nijst P, Verbrugge FH, et al. Impact of iron deficiency on exercise capacity and outcome in heart failure with reduced, mid-range and preserved ejection fraction. Acta Cardiol 2018;73:115-23.

88. Anker SD, Kirwan BA3, van Veldhuisen DJ,. et al. Effects of ferric carboxymaltose on hospitalisations and mortality rates in iron-deficient heart failure patients: an individual patient data meta-analysis. Eur J Heart Fail 2018;201:125-33.

89. Okonko DO, Grzeslo A, Witkowski T, et al. Effect of intravenous iron sucrose on exercise tolerance in anemic and nonanemic patients with symptomatic chronic heart failure and iron deficiency FERRIC-HF: a randomized, controlled, observer-blinded trial. J Am Coll Cardiol 2008;51:103-12.

90. Jankowska EA, Tkaczyszyn M, Suchocki T, et al. Effects of intravenous iron therapy in iron-deficient patients with systolic heart failure: a meta-analysis of randomized controlled trials. Eur J Heart Fail 2016;18:786-95.

91. Caravita S, Faini A, Vignati C, et al. Effects of intravenous iron therapy on chemoreflex sensitivity and sleep disordered breathing in chronic heart failure (abs). Eur Heart J 2017;38(s1):ehx502.P2425.

92. Agarwal R, Vasavada N, Sachs NG, Chase S. Oxidative stress and renal injury with intravenous iron in patients with chronic kidney disease. Kidney Int 2004;65:2279-89.

93. Van Buren P, Velez RL, Vaziri ND, Zhou XJ. Iron overdose: a contributor to adverse outcomes in randomized trials of anemia correction in CKD. Int Urol Nephrol 2012;44:499-507.

94. Klip IT, Comin-Colet J, Voors AA, et al. Iron deficiency in chronic heart failure: an international pooled analysis. Am Heart J 2013;165:575-82.

95. Comin-Colet J, Enjuanes C, González G, et al. Iron deficiency is a key determinant of health-related quality of life in patients with chronic heart failure regardless of anaemia status. Eur J Heart Fail 2013;15:1164-72.

96. Pozzo J, Fournier P, Delmas C, et al. Absolute iron deficiency without anaemia in patients with chronic systolic heart failure is associated with poorer functional capacity. Arch Cardiovasc Dis 2017;110:99-105. 